ELECTRONIC RESEARCH ANNOUNCEMENTS OF THE AMERICAN MATHEMATICAL SOCIETY

Volume 5, Pages 35-39 (April 1, 1999)

S $1079-6762(99) 00058-\mathrm{X}$

\title{
THE SPECIAL SCHUBERT CALCULUS IS REAL
}

\author{
FRANK SOTTILE
}

(Communicated by Robert Lazarsfeld)

\begin{abstract}
We show that the Schubert calculus of enumerative geometry is real, for special Schubert conditions. That is, for any such enumerative problem, there exist real conditions for which all the a priori complex solutions are real.
\end{abstract}

Fulton asked how many solutions to a problem of enumerative geometry can be real, when that problem is one of counting geometric figures of some kind having specified position with respect to some general fixed figures [5]. For the problem of plane conics tangent to five general conics, the (surprising) answer is that all 3264 may be real [10]. Recently, Dietmaier has shown that all 40 positions of the Stewart platform in robotics may be real [2]. Similarly, given any problem of enumerating lines in projective space incident on some general fixed linear subspaces, there are real fixed subspaces such that each of the (finitely many) incident lines are real [13]. Other examples are shown in [12, 14], and the case of 462 4-planes meeting 12 general 3-planes in $\mathbb{R}^{7}$ is due to an heroic symbolic computation [4].

For any problem of enumerating $p$-planes having excess intersection with a collection of fixed planes, we show there is a choice of fixed planes osculating a rational normal curve at real points so that each of the resulting $p$-planes is real. This has implications for the problem of placing real poles in linear systems theory [1] and is a special case of a far-reaching conjecture of Shapiro and Shapiro [15].

\section{Special Schubert CONDitions}

For background on the Grassmannian, Schubert cycles, and the Schubert calculus, see any of $[8,7,6]$. Let $m, p \geq 1$ be integers. Let $\gamma$ be a rational normal curve in $\mathbb{R}^{m+p}$. For $k>0$ and $s \in \gamma$, let $K_{k}(s)$ be the $k$-plane osculating $\gamma$ at $s$. For every integer $a>0$, let $\tau_{a}(s)$ be the special Schubert cycle consisting of $p$ planes $H$ in $\mathbb{C}^{m+p}$ which meet $K_{m+1-a}(s)$ nontrivially, and let $\tau^{a}(s)$ be the special Schubert cycle consisting of $p$-planes $H$ in $\mathbb{C}^{m+p}$ meeting $K_{m-1+a}(s)$ improperly: $\operatorname{dim} H \cap K_{m-1+a}(s)>a-1$. These cycles $\tau_{a}(s)$ and $\tau^{a}(s)$ each have codimension $a$ and $\tau^{1}=\tau_{1}$. Recall that the Grassmannian of $p$-planes in $\mathbb{C}^{m+p}$ has dimension

Received by the editors December 20, 1998.

1991 Mathematics Subject Classification. Primary 14P99, 14N10, 14M15, 14Q20; Secondary $93 \mathrm{~B} 55$.

Key words and phrases. Schubert calculus, enumerative geometry, Grassmannian, pole placement problem.

MSRI preprint \# 1998-067.

Research supported by NSF grant DMS-9701755.

(C)1999 American Mathematical Society 
$m p$. For any Schubert condition $w$, let $\sigma_{w}(s)$ be the Schubert cycle of type $w$ given by the flag osculating $\gamma$ at $s$ and set $|w|$ to be the codimension of $\sigma_{w}(s)$.

Theorem 1. Let $a_{1}, \ldots, a_{n}$ be positive integers with $a_{1}+\cdots+a_{n}=m p$. For each $i=1, \ldots, n$ let $\sigma_{i}(s)$ be either $\tau^{a_{i}}(s)$ or $\tau_{a_{i}}(s)$. Then there exist real points $0, \infty, s_{1}, \ldots, s_{n} \in \gamma$ such that for any Schubert conditions $w, v$, and integer $k$ with $|w|+|v|+a_{k}+\cdots+a_{n}=m p$, the intersection

$$
\sigma_{w}(0) \cap \sigma_{v}(\infty) \cap \sigma_{k}\left(s_{k}\right) \cap \cdots \cap \sigma_{n}\left(s_{n}\right)
$$

is transverse with all points of intersection real.

Our proof is inspired by the Pieri homotopy algorithm of [9].

We prove this in the case that each $\sigma_{i}(s)=\tau_{a_{i}}(s)$. This is no loss of generality, as the cycles $\tau_{a}(s)$ and $\tau^{a}(s)$ share the properties we need. We use the following two results of Eisenbud and Harris [3], who studied such intersections in their theory of limit linear systems. For a Schubert class $w$, let $w * a$ be the index of summation in the Pieri formula in the cohomology of the Grassmannian [6],

$$
\sigma_{w} \cdot \tau_{a}=\sum_{v \in w * a} \sigma_{v}
$$

\section{Proposition 2.}

1. (Theorem 2.3 of [3]) Let $s_{1}, \ldots, s_{n}$ be distinct points on $\gamma$ and $w_{1}, \ldots, w_{n}$ be Schubert conditions. Then the intersection of Schubert cycles

$$
\sigma_{w_{1}}\left(s_{1}\right) \cap \sigma_{w_{2}}\left(s_{2}\right) \cap \cdots \cap \sigma_{w_{n}}\left(s_{n}\right)
$$

is proper in that it has dimension $m p-\left|w_{1}\right|-\cdots-\left|w_{n}\right|$.

2. (Theorem 8.1 of [3]) For any Schubert condition $w$, integer $a>0$, and $0 \in \gamma$, we have

$$
\lim _{t \rightarrow 0}\left(\sigma_{w}(0) \cap \tau_{a}(t)\right)=\bigcup_{v \in w * a} \sigma_{v}(0),
$$

the limit taken along the rational normal curve, and as schemes.

Proof of Theorem 1. We argue by downward induction on $k$. The initial case of $k=n$ holds as Pieri's formula implies the intersection is a single, necessarily real, point. Suppose it holds for $k$, and let $w, v$ satisfy $|w|+|v|+a_{k-1}+\cdots+a_{n}=m p$.

Claim. The cycle

$$
\sum_{u \in w * a_{k-1}} \sigma_{u}(0) \cap \sigma_{v}(\infty) \cap \tau_{a_{k}}\left(s_{k}\right) \cap \cdots \cap \tau_{a_{n}}\left(s_{n}\right)
$$

is free of multiplicities.

If not, then two summands, say $u$ and $u^{\prime}$, have a point in common and so

$$
\sigma_{u}(0) \cap \sigma_{u^{\prime}}(0) \cap \sigma_{v}(\infty) \cap \tau_{a_{k}}\left(s_{k}\right) \cap \cdots \cap \tau_{a_{n}}\left(s_{n}\right)
$$

is nonempty. However, $\sigma_{u}(0) \cap \sigma_{u^{\prime}}(0)$ is a Schubert cycle of smaller dimension. Thus the intersection (2) must be empty, by Proposition 2 (1).

From the claim and Proposition $2(2)$, there is an $\epsilon_{w, v}>0$ such that if $0<t \leq$ $\epsilon_{w, v}$, then

$$
\sigma_{w}(0) \cap \sigma_{v}(\infty) \cap \tau_{a_{k-1}}(t) \cap \tau_{a_{k}}\left(s_{k}\right) \cap \cdots \cap \tau_{a_{n}}\left(s_{n}\right)
$$

is transverse with all points of intersection real. Set

$$
s_{k+1}:=\min \left\{\epsilon_{w, v}|m p=| w|+| v \mid+a_{k-1}+\cdots+a_{n}\right\} .
$$


Remark. Eisenbud and Harris [3] prove Proposition 2 (2) for any nondegenerate $\operatorname{arc} \gamma$. Theorem 1 may be similarly strengthened.

\section{CONSEQUences}

Since small real perturbations of the points $s_{1}, \ldots, s_{n}$ cannot create or destroy real points in a transverse intersection, Theorem 1 has the following consequence.

Corollary 3. There is an open subset (in the classical topology on $\gamma^{n}$ ) consisting of $n$-tuples $\left(s_{1}, \ldots, s_{n}\right) \in \gamma^{n}$ such that (1) is transverse with all points of intersection real.

Theorem 1 proves part of a conjecture of Shapiro and Shapiro.

Conjecture 4 (Shapiro and Shapiro). Let $m, p \geq 1$ and $w_{1}, \ldots, w_{n}$ be Schubert conditions on $p$-planes in $(m+p)$-space. If $s_{1}, \ldots, s_{n} \in \gamma$ are real points so that

$$
\sigma_{w_{1}}\left(s_{1}\right) \cap \sigma_{w_{2}}\left(s_{2}\right) \cap \cdots \cap \sigma_{w_{n}}\left(s_{n}\right)
$$

is zero-dimensional, then all points of intersection are real.

When the points $s_{1}, \ldots, s_{n}$ are distinct and $\left|w_{1}\right|+\cdots+\left|w_{n}\right|=m p,(3)$ is zerodimensional [3]. Besides Theorem 1, there is substantial and compelling evidence for the validity of Conjecture $4[4,16,11,15]$. In every instance (choice of $w_{1}, \ldots, w_{n}$ and distinct real points $s_{1}, \ldots, s_{n} \in \gamma$ ) checked, all points in (3) are real. This includes some when the $w_{i}$ are not special Schubert conditions [15] and some spectacular computations $[4,16]$. For some sets of Schubert conditions, we have proven that for every choice of distinct real points $s_{1}, \ldots, s_{n} \in \gamma$, all points in (3) are real. In every known case, the intersection scheme is reduced when the parameters $s_{1}, \ldots, s_{n}$ are distinct and real. We conjecture this is always the case.

Conjecture 5. Let $m, p \geq 1$ and $w_{1}, \ldots, w_{n}$ be Schubert conditions with $m p=$ $\left|w_{1}\right|+\cdots+\left|w_{n}\right|$. If $s_{1}, \ldots, s_{n} \in \gamma$ are distinct and real, then the intersection scheme (3) is reduced.

The number of real points in the scheme (3) is locally constant on the set of parameters for which it is reduced. Thus for special Schubert conditions, Conjecture 5 would imply Conjecture 4, by Theorem 1 . In fact, more is true.

Theorem 6. Conjecture 5 implies Conjecture 4.

Proof. By Remark 3.4 to Theorem 3.3 of [15], if Conjecture 4 holds when $a_{1}=\cdots=$ $a_{m p}=1$, then it holds for any collection of Schubert conditions, if the cycles (3) are reduced for any choice of $w_{1}, \ldots, w_{n}$ with $m p=\left|w_{1}\right|+\cdots+\left|w_{n}\right|$ and general $s_{1}, \ldots, s_{n} \in \gamma$. Both of these conditions are supplied by Conjecture 5 .

When $n=m p$ so that $a_{1}=\cdots=a_{m p}=1,(1)$ is a special case of the pole placement problem in systems theory [1]. A physical system (e.g. a mechanical linkage), called a plant with $m$ inputs and $p$ measured outputs, whose evolution is governed by a system of linear differential equations, is modeled by a $m \times(m+p)$ matrix of univariate polynomials $[N(s): D(s)]$. The largest degree of a maximal minor of this matrix is the MacMillan degree, $n$, of the evolution equation. For $s_{1}, \ldots, s_{n} \in \mathbb{C}$, any $p$-plane $H$ satisfying

$$
H \cap \text { row } \operatorname{span}\left[N\left(s_{i}\right): D\left(s_{i}\right)\right] \neq\{0\}, \quad i=1, \ldots, n,
$$


gives a constant linear feedback law for which the resulting closed system has natural frequencies (poles) $s_{1}, \ldots, s_{n}$.

In this way, the pencil $K(s)$ of $m$-planes osculating $\gamma$ represents a particular $m$-input $p$-output plant of McMillan degree $m p$, and the $p$-planes in

$$
\tau_{1}\left(s_{1}\right) \cap \tau_{1}\left(s_{2}\right) \cap \cdots \cap \tau_{1}\left(s_{m p}\right)
$$

represent linear feedback laws for which the resulting closed system has natural frequencies $s_{1}, \ldots, s_{m p}$. Since translation along $\gamma$ fixes the system and acts on the feedback laws by a real linear transformation, we may assume that $s_{1}, \ldots, s_{m p}<0$ and so we obtain the existence of a stable system with only real feedback laws.

Corollary 7. The plant represented by $K(s)$ can be stabilized by real feedback laws in such a way that all feedback laws are real.

\section{REFERENCES}

[1] C. I. Byrnes, Pole assignment by output feedback, in Three Decades of Mathematical Systems Theory, H. Nijmeijer and J. M. Schumacher, eds., vol. 135 of Lecture Notes in Control and Inform. Sci., Springer-Verlag, Berlin, 1989, pp. 31-78. MR 90k:93001

[2] P. Dietmaier, The Stewart-Gough platform of general geometry can have 40 real postures, in Advances in Robot Kinematics: Analysis and Control, Kluwer Academic Publishers, 1998, pp. 1-10. CMP 99:01

[3] D. Eisenbud And J. Harris, Divisors on general curves and cuspidal rational curves, Invent. Math., 74 (1983), pp. 371-418. MR 85h:14019

[4] J.-C. Faugère, F. Roulllier, and P. Zimmermann, Private communication. 1998.

[5] W. Fulton, Introduction to Intersection Theory in Algebraic Geometry, CBMS 54, AMS, 1984. MR 85j: 14008

[6] — - Young Tableaux, Cambridge University Press, 1997. CMP 97:16

[7] P. Griffiths and J. Harris, Principles of Algebraic Geometry, J. Wiley and Sons, 1978. MR 80b:14001

[8] W. V. D. Hodge and D. Pedoe, Methods of Algebraic Geometry, vol. II, Cambridge University Press, 1952. MR 13:972c

[9] B. Huber, F. Sottile, and B. Sturmfels, Numerical Schubert calculus. J. Symb. Comp., 26 (1998), pp. 767-788. CMP 99:06

[10] F. Ronga, A. Tognoli, And T. Vust, The number of conics tangent to 5 given conics: the real case, Rev. Mat. Univ. Complut. Madrid, 10 (1997), pp. 391-421. MR 99d:14059

[11] J. Rosenthal And F. Sottile, Some remarks on real and complex output feedback, Systems \& Control Lett., 33 (1998), pp. 73-80. MR 98m:93052. For a description of the computational aspects, see http://www.nd.edu/ ${ }^{\sim}$ rosen/pole/.

[12] F. SotriLE, Enumerative geometry for real varieties, in Algebraic Geometry, Santa Cruz 1995, J. Kollár, R. Lazarsfeld, and D. Morrison, eds., vol. 62, Part 1 of Proc. Sympos. Pure Math., Amer. Math. Soc., 1997, pp. 435-447. CMP 98:07

[13] — Enumerative geometry for the real Grassmannian of lines in projective space, Duke Math. J., 87 (1997), pp. 59-85. MR 99a:14079

[14] — Real enumerative geometry and effective algebraic equivalence, J. Pure Appl. Alg., 117 \& 118 (1997), pp. 601-615. Proc., MEGA'96. MR 98h:14064

[15] - Real Schubert calculus: Polynomial systems and a conjecture of Shapiro and Shapiro. MSRI preprint \# 1998-066. For an archive of computations and computer algebra scripts, see http://www.math.wisc.edu/ sottile/pages/shapiro/index.html, 1998.

[16] J. VERSCHELDE, Numerical evidence of a conjecture in real algebraic geometry. MSRI preprint \# 1998-064, 1998. 
Mathematical Sciences Research Institute, 1000 Centennial Drive, Berkeley, CA 94720

Current address: Department of Mathematics, University of Wisconsin, Van Vleck Hall, 480 Lincoln Drive, Madison, Wisconsin 53706-1388

$U R L:$ http://www.math.wisc.edu/ ${ }^{\sim}$ sottile

E-mail address: sottile@math.wisc.edu 